\title{
AN INTERFEROMETRIC PROCESSING METHOD TO MAINTAIN BOTH ACCURACY AND RESOLUTION OF DEM USING GAOFEN-3 SAR DATA
}

\author{
Tao Li, Xinming Tang, Xiaoming Gao, Qianfu Chen, Xiang Zhang, Li Guo
}

Satellite Surveying and Mapping Application Centre, National Administration of Surveying, Mapping and Geo-information, Beijing 100048, China - (lit, txm, gaoxm, chenqf, zhangx, guol)@sasmac.cn

\section{Commission III, WG III/3}

KEY WORDS: Gaofen-3, TanDEM-X, DEM, multi-look

\begin{abstract}
:
Gaofen-3 (GF-3) is the only in-orbit SAR satellite of China civilian fields. It is designed especially for ocean observation but not for interferometric applications. However, during the past one and a half years, the orbits of the satellite were adjusted for several times to ensure that the perpendicular baseline is short enough to provide interferograms. In this paper, we used the multi-look iteration algorithm to analyze the DEM obtained from InSAR. We first provide the theory of the method by considering the relationship between multi-look factor and the interferometric phase gradient. Then the GF-3 data as well as TanDEM-X data covering Songshan Mountain are taken for experiments. We use both the GCP data as well as the SRTM DEM for reference. The root-mean-square (RMS) values of TanDEM-X DEM assessed using GCP are $9.4 \mathrm{~m}, 9.3 \mathrm{~m}$ and $8.3 \mathrm{~m}$ with reference to ML factors of $8 \times 8,4 \times 4$ and $2 \times 2$, respectively. If we assess using SRTM, the corresponding RMS are $1.7 \mathrm{~m}, 5.4 \mathrm{~m}$ and $5.4 \mathrm{~m}$. The result is opposite to that obtained using GCP given that the grid size of SRTM DEM is $90 \mathrm{~m}$. The larger the ML factor, the more similar the calculated DEM to SRTM. RMS of GF-3 DEM compared to GCP is $10.2 \mathrm{~m}, 13.0 \mathrm{~m}$ and $13.8 \mathrm{~m}$ with reference to ML factors of $8 \times 8,4 \times 4$ and $2 \times 2$, respectively. While that compared to SRTM is $4.6 \mathrm{~m}, 15.1 \mathrm{~m}$ and $23.7 \mathrm{~m}$. The accuracy is low compared to TanDEM-X DEM. Results show that the GF-3 data is potential in providing DEM data. However, the operational applications using GF-3 as interferometric data source would be challenging because of the instability of baseline coherence as well as the temporal coherence.
\end{abstract}

\section{INTRODUCTION}

InSAR is a powerful tool to provide global digital elevation model (DEM) given its high sensitivity to terrain undulation. The famous InSAR DEM acquisition missions are shuttle radar topography mission (SRTM) and TanDEM-X mission. SRTM is designed by following DTED standard, the absolute height accuracy is expected to be $16 \mathrm{~m}$. However, the final accuracy is $9.0 \mathrm{~m}$ relative to the ground control points (GCP) (Rodńguez et al., 2005). TanDEM-X perform even better accuracy after elaborate processing. The HRTI-3 standard applied in TanDEM-X mission specify that the absolute height accuracy should be better than $10 \mathrm{~m}$. The final accuracy after raw DEM block adjustment is $1.3 \mathrm{~m}$ if the worst DEM data in the Antarctica are not included in statistics (Buckreuß, 2016).

Accuracy of the DEM is influenced by three kinds of errors. The first is geometric error including the position and velocity of the satellite, the slant range, the Doppler centroid error. The second error source is related to the baseline of which the accuracy is acquired to be better than $1 \mathrm{~mm}$ (Montenbruck et al., 2011). After in-orbit calibration, the baseline accuracy is found to be $2-10 \mathrm{~mm}$, which is less than expected (Hueso González et al., 2012). However, through the baseline calibration and raw DEM block adjustment procedures, the error related to baseline is suppressed and the accuracy is apparently improved. The final problem is the interferometric phase which is difficult to determine. The phase error, excluding the absolute phase offset which is calculated using radargrammetry (Rossi et al., 2012), should be small enough given its high influence on height error. If the height of ambiguity (HoA) is $35-55 \mathrm{~m}$, then $10^{\circ}$ phase error induces $0.97-1.53 \mathrm{~m}$ height error. Phase unwrapping is one of the most important phase-related error source.

The phase unwrapping algorithm depends on the phase gradient. Relationship between them is not determinative. The higher the gradient, the more chance to get frustrated results (Jiang et al., 2011). Multi looking (ML) is effective to decrease the phase gradient of signal noise, but the useful information related to deformation and elevation are all degraded. If we use a small ML factor, the resolution is ensured but the phase noise cannot be decreased, making it challenging to unwrap the phase correctly. Therefore, in this study, an improved method to maintain both the accuracy and resolution of DEM using InSAR data is used (Gao et al., 2017).

The detectable phase gradient (DPG) of InSAR processing is expressed using the pixel spacing and the ML factor. The more the ML factor, the less the minimum DPG, and vice versa. When the minimum DPG is less than 0 , we define the corresponding ML factor as the maxima. The phase gradient consists of two parts. The first part is the spatially lowfrequency component such as terrain information as well as atmospheric phase screen. Given that the atmospheric phase is self-correlated within tens of kilometres and affects the final phase gradient slightly, we do not model the atmospheric phase gradient. The terrain phase gradient is well described by fractional Brownian motion ( $\mathrm{fBm})$ model. Through the modelling and analysis, we find that the ML factor should be as small as possible to reduce the phase standard deviation. The second part is the spatially high-frequency component such as noise. The noise phase gradient is expressed using Gaussian 
hypergeometric function which is related to the coherence and ML factor. Probability density function (PDF) show that the ML factor should be kept as large as possible to decrease the standard deviation. The noise phase gradient increases as the ML factor decreases. When ML factor reaches its minima, the phase noise gradient is greater than the maximum DPG, making the phase gradient unable to contain any other phase gradient related to elevation.

In this paper, we overview the fundamental of the relationship between DPG and ML factor in Chapter 2. After that, the proposed method is applied in GF-3 interferograms in Chapter 3. Some conclusions are drawn in Chapter 4.

\section{METHODOLOGY}

\subsection{Components of interferometric phase}

The interferometric phase mainly consists of five components. They are the phase related to flat earth, atmosphere, deformation, noise and terrain. The flattening phase is calculated using external DEM data such as SRTM. The atmospheric phase is not modelled due to its highly spatial selfcorrelation character. If we use the GF-3 data, the deformation should be avoided due to its non-negligible influence on interferometric phase. We choose the Songshan mountain region where the deformation can be barely observed. Therefore, we can distinguish noise and terrain phase from others. The interferometric phase after processing is expressed using

$$
\varphi=\varphi_{\text {top }}+\varphi_{\text {noi }}
$$

where $\varphi$ is the unwrapped interferometric phase, $\varphi_{\text {top }}$ is the topographic phase, $\varphi_{\text {noi }}$ is the noise phase.

Given that the phase gradient is calculated from interferometric phase, we can model the phase gradient using the same way, i.e.,

$$
\Delta \varphi=\Delta \varphi_{\text {top }}+\Delta \varphi_{\text {noi }},
$$

where the three components of the equation refer to the interferometric phase gradient, the topographic phase gradient and the noise phase gradient, respectively.

\subsection{Interferometric phase gradient and ML factor}

Limitation of the interferometric phase gradient (IPG) is $\pi$ according to the Shannon's sampling theorem. We expect the IPG far less than $\pi$ because the phase unwrapping accuracy is dependent on the IPG. Given the equation provided by Jiang (Jiang et al., 2011), we can express the DPG of C-band interferogram using

$$
\left\{\begin{array}{l}
\Delta \varphi_{(\gamma, N)}^{\min }=(0.000824961 \times \mu(M)-0.0551983) \times(\gamma-1)+2.23 \times 10^{-4} \\
\Delta \varphi_{(\gamma, N)}^{\max }=\frac{\pi}{\mu(M)}+\left(8.10676 \times \mu(M)^{-1.258}\right) \times(\gamma-1)
\end{array}\right.
$$

where $\Delta \varphi_{(\gamma, N)}^{\min }$ and $\Delta \varphi_{(\gamma, N)}^{\max }$ are the minimum and maximum DPG, $\mu(M)$ is the smaller value of pixel spacing between range and azimuth directions after multi-looking processing using ML factor of $M, \gamma$ is the coherence. The coherence ranges from 0 to 1. We take the ML factor that makes the maximum DPG and minimum DPG equal as the basis to conduct phase unwrapping. Take GF-3 for example, the pixel spacing is around $3 \mathrm{~m}$, if the ML factor is 1,4 , and 16 , then the corresponding coherence must be greater than $0.50,0.35$ and 0.19 . If the coherence is 0 , the phase provides no useful information. The ML factor values greater than 23 allow us to detect all the phase gradient greater than 0 . The corresponding parameter with TanDEM-X is 66 , meaning that the maximum ML factor adopted in TanDEM-X processing can be $8 \times 8$, ML factors greater than this barely improve the interferometric quality. For comparison purpose, we use the maximum ML factor of $8 \times 8$ for both GF- 3 and TanDEM-X data.

\subsection{Topographic phase gradient and ML factor}

Topography is described using $\mathrm{fBm}$, the PDF of phase gradient is then expressed using

$$
\begin{gathered}
p d f\left(\Delta h^{a}\right)=\frac{C_{a h}}{\sigma|d a|^{H}} e^{\left(-\frac{\left(\Delta \Delta^{a}\right)^{2}}{2 \sigma^{2}(d a)^{2 H}}\right)}, \\
p d f\left(\Delta h^{r}\right)=\frac{C_{r h}}{\sigma|d r|^{H}} e^{\left(-\frac{\left(\Delta h^{r}\right)^{2}}{2 \sigma^{2}(d r)^{2 H}}\right)},
\end{gathered}
$$

where

$$
\begin{gathered}
d_{a}=\sqrt{\mu_{a}+\left(\frac{\Delta h^{a}}{\tan \theta}\right)^{2}}, \\
d_{r}=\mu_{r}+\frac{\Delta h^{r}}{\tan \theta} .
\end{gathered}
$$

In the above equations, we use the incidence angle $(\theta)$, the normalizing constants $\left(c_{a h}\right.$ and $\left.c_{r h}\right)$, and the Hurst parameter $(H)$ to express the characterization of the topographic phase gradient. Although the terrain information distributes similarly in both east-west and south-north directions, the side-looking of SAR payload changes the east-west topographic phase to skew $\mathrm{fBm}$ as shown in Equ. (6). Both $\mathrm{fBm}$ and skew fBm show the similar mathematical feature, i.e., the smaller the ML factor, the more centralized the data. Therefore, we must adopt the small ML factor to maintain the consistent topographic phase gradient.

\subsection{Noise phase gradient and ML factor}

Interferometric noise phase is expressed using a Gauss Hypergeometric Function such as

$$
\begin{gathered}
p d f\left(\varphi ; \gamma, M, \varphi_{0}\right)=\frac{\left(1-|\gamma|^{2}\right)^{M}}{2 \pi} \times\left\{\frac{\Gamma(2 M-1)}{\left.\Gamma(M)\right|^{2} 2^{2(M-1)}}\left[\frac{(2 M-1) \beta}{\left(1-\beta^{2}\right)^{M+0.5}}\left(\frac{\pi}{2}+a c r \sin \beta+\frac{1}{\left(1-\beta^{2}\right)^{M}}\right)\right],\right. \\
\left.+\frac{1}{2(M-1)} \sum_{k=0}^{M-2} \frac{\Gamma(M-0.5)}{\Gamma(M-0.5-k)} \frac{\Gamma(M-1-k)}{\Gamma(M-1)} \frac{1+(2 k+1) \beta^{2}}{\left(1-\beta^{2}\right)^{k+2}}\right\},(M>1)
\end{gathered}
$$

where $\beta=|\gamma| \cos \left(\varphi-\varphi_{0}\right), \Gamma$ is the GAMMA function. If the noise of two points follows the same Gauss hypergeometric distribution and they are independent from each other, the PDF of phase gradient between the two points are expressed as

$$
p d f\left(\Delta \varphi_{n o i}\right)=\int_{-\pi}^{\pi} p d f\left(\varphi ; \gamma, N, \varphi_{0}\right) w\left[p d f\left(\varphi+\Delta \varphi_{n o i} ; \gamma, N, \varphi_{0}\right)\right] d \varphi .
$$


where $w(\cdot)$ is the phase unwrapping operator.

Opposite conclusion is drawn if we compare Equ. (9) to (4) (7). The ML factor is expected to be as great as possible to suppress the noise phase. A greater ML factor ensures that the noise data distribute more centralized. Therefore, the noise phase gradient is more uniform, and the phase unwrapping procedure is more likely to be successfully conducted.

During InSAR processing chains, we need to keep the maximum DPG greater than the noise phase gradient. The corresponding ML factor should be greater than 4 which is calculated using the equations (3) and (9).

\subsection{Interferometric processing using different ML factors}

Maximum and minimum ML factors are $8 \times 8$ and $2 \times 2$ according to our modeling. Therefore, we need to use different ML factors to balance the contradiction between noise phase gradient and terrain phase gradient. We first calculate a coarse DEM using ML factor of 64 . The noise phase is decreased during the processing. But the terrain-related phase is more possible to be skewed, making the corresponding coherence too low to be unwrapped. We mask the coarse DEM using coherence threshold to avoid potential phase unwrapping error. Then the coarse DEM with leaks are interpolated and uses as reference to calculate DEM with ML factor of $4 \times 4$. Although the noise phase gradient increases, the elevation phase gradient decreases due to the reference DEM. The same processing method is applied to obtain the final DEM with ML factor of $2 \times 2$. The accuracy of the final DEM is high because the phase unwrapping accuracy is ensured by decreasing the phase gradient. Meanwhile, the resolution of the final DEM reaches as high as twice of the pixel spacing size.

\section{EXPERIMENTS AND RESULTS}

\subsection{Data selection}

The data covering Songshan Mountain is selected given that the elevation changes fast in the region (Fig. 1). Phase unwrapping is a challenging task in the experimental region.

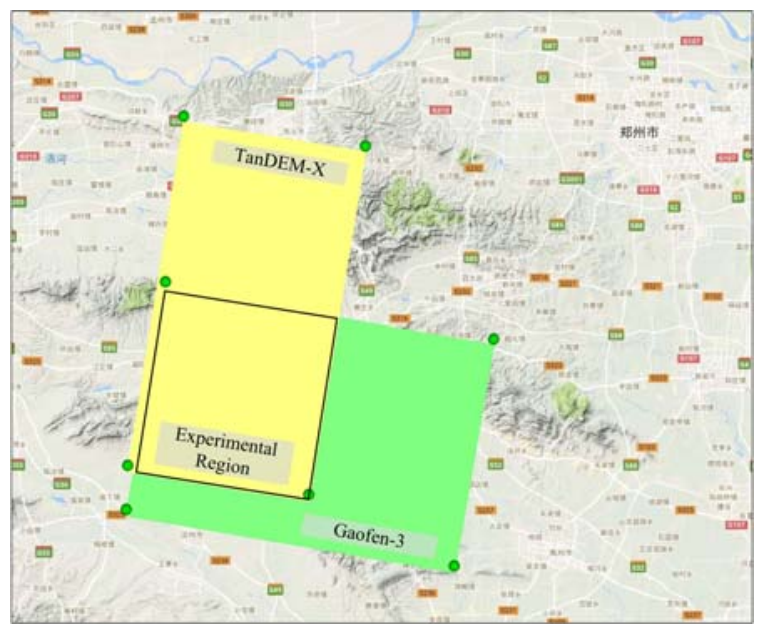

Figure 1. Image coverage of the selected data. The yellow region is TanDEM-X image, the green region is Gaofen-3 image. While the black rectangle is the selected experimental area.
The TanDEM-X image pair is taken on 20140125 (YYYYMMDD). The range pixel spacing is $1.36 \mathrm{~m}$, azimuth pixel spacing is $2.05 \mathrm{~m}$. Original coverage of the image is 30 $\mathrm{km} \times 50 \mathrm{~km}$. The GF-3 image pair is taken by considering the interferometric precondition. One of the main parameter to control is the recursive baseline. It should be less than the critical baseline. In our experiment, the critical baseline is $10576 \mathrm{~m}$ in flat region. Perpendicular baseline of the selected image pair is $980 \mathrm{~m}$. The master image is taken on 20161228 . The slave image is taken on 20161129. The range and azimuth pixel spacings are 2.25 and $2.86 \mathrm{~m}$, respectively. Original coverage of the image is $55 \mathrm{~km} \times 62 \mathrm{~km}$. We choose the common coverage of the two image pairs. The selected area is $30 \mathrm{~km} \times 24 \mathrm{~km}$

\subsection{Experimental results and analysis}

DEMs obtained from GF-3 and TanDEM-X are assessed using SRTM data, ICESat data as well as ground control points. The ground control points are obtained by following the 1:10000 measurement scale. Accuracy values are listed in Tab. 1 and Tab. 2.

GF-3 DEMs are worse than TanDEM-X DEMs given that the temporal coherence induced by the temporal baseline is not able to be avoided. Meanwhile, the interferometric parameters, such as baseline length and geometric locations, are not as high as those stated by TanDEM-X. Accuracy values relative to SRTM data are 4.64 and $1.68 \mathrm{~m}$ for GF-3 DEM and TanDEM-X DEM when ML factor is 64 . If the ML factor is only 4 , which is the minimum for phase unwrapping, the accuracy of GF-3 can be as low as $13.84 \mathrm{~m}$ assessed using GCP. The final accuracy of TanDEM-X is $8.31 \mathrm{~m}$, which is $5.5 \mathrm{~m}$ better than GF-3.

When assessing using ICESat and GCP data, we find obvious accuracy discrepancy compared to SRTM results. The three datasets are used for different purpose. The SRTM data are the most comprehensive, the assessment results indicate the systematic error, while the random error is suppressed during statistics. Therefore, the error is the smallest if most of the errors are randomly distributed. Which is the truth for TanDEM-X DEM error. The ICESat and GCP data are used to assess the final quality of the DEM. They are sensitive to gross error which may occur in the decoherent regions. The height accuracy of the ICESat data is within $0.15 \mathrm{~m}$, while that of the GCP is centimetric. If the gross errors exist in the TanDEM-X DEM, the two indicators increase accordingly.

\begin{tabular}{|l|l|l|l|}
\hline & $8 \times 8$ & $4 \times 4$ & $2 \times 2$ \\
\hline SRTM & 4.64 & 15.13 & 23.66 \\
\hline ICESat & 6.32 & 11.47 & 13.37 \\
\hline GCP & 10.22 & 12.98 & 13.84 \\
\hline
\end{tabular}

Table 1. Gaofen-3 DEM accuracy assessed using SRTM, ICESat and GCP

\begin{tabular}{|l|l|l|l|}
\hline & $8 \times 8$ & $4 \times 4$ & $2 \times 2$ \\
\hline SRTM & 1.68 & 5.36 & 5.43 \\
\hline ICESat & 5.38 & 5.34 & 4.91 \\
\hline GCP & 9.42 & 9.29 & 8.31 \\
\hline
\end{tabular}

Table 2. TanDEM-X DEM accuracy assessed using SRTM, ICESat and GCP 
DEMs obtained from GF-3 are shown in Fig. (2). That obtained from TanDEM are shown in Fig. (3). (a) - (c) are DEMs calculated using ML factors of $8 \times 8,4 \times 4$ and $2 \times 2$. Coherence of GF-3 is low compared to TanDEM-X, especially in the flat regions covered by vegetations, i.e., rectangle areas in the Fig. 2 The proposed method is expected to be better and better when the ML factors are smaller and smaller. However, in the flat areas, the decrease of the noise phase gradient in the flat area is less than the increase of the noise induced by smaller ML factors. While the expected results are observed in the circular regions of Fig. 2, the terrain becomes smooth when ML factors decrease, proving the availability of the method. But the accuracy of the specified region is low, that is because of the error propagation in the phase unwrapping procedure.
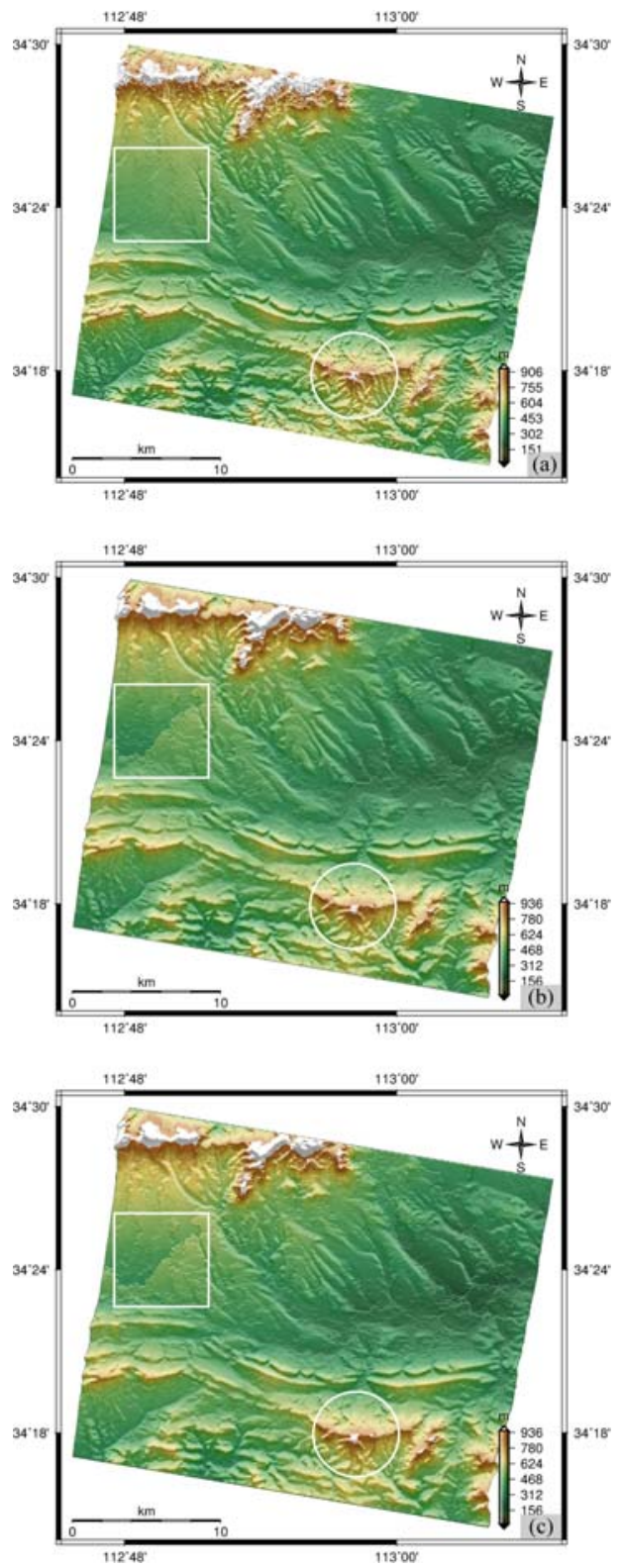

Figure 2. Gaofen-3 DEMs obtained using ML factors of (a) $8 \times 8$, (b) $4 \times 4$, and (c) $2 \times 2$. Rectangles are flat areas. Circles are the highest region named Laopozhai.

DEMs calculated using TanDEM-X are shown in Fig. 3. The terrain is consistent all over the image even with ML factor of 4 .
The coherence is high enough by eliminating the temporal baseline. More details are revealed when ML factors decrease. In the elliptical area where residential area is located, no texture is provided in (a), but the house is observed in (c). RMS with referent to SRTM DEM show that the quality of the DEM decreases when ML factors decrease. That is because the smaller the ML factor, the higher the resolution of DEM. The final resolution of the DEM is around $4 \mathrm{~m} \times 4 \mathrm{~m}$, while the resolution of SRTM is $90 \mathrm{~m}$. Differences between the data increase when ML factors decrease. The accuracy assessed using ICESat and GCP are different from SRTM. The DEM with smallest ML factor is the best. Therefore, the proposed method can be used to maintain both accuracy and resolution of DEM.

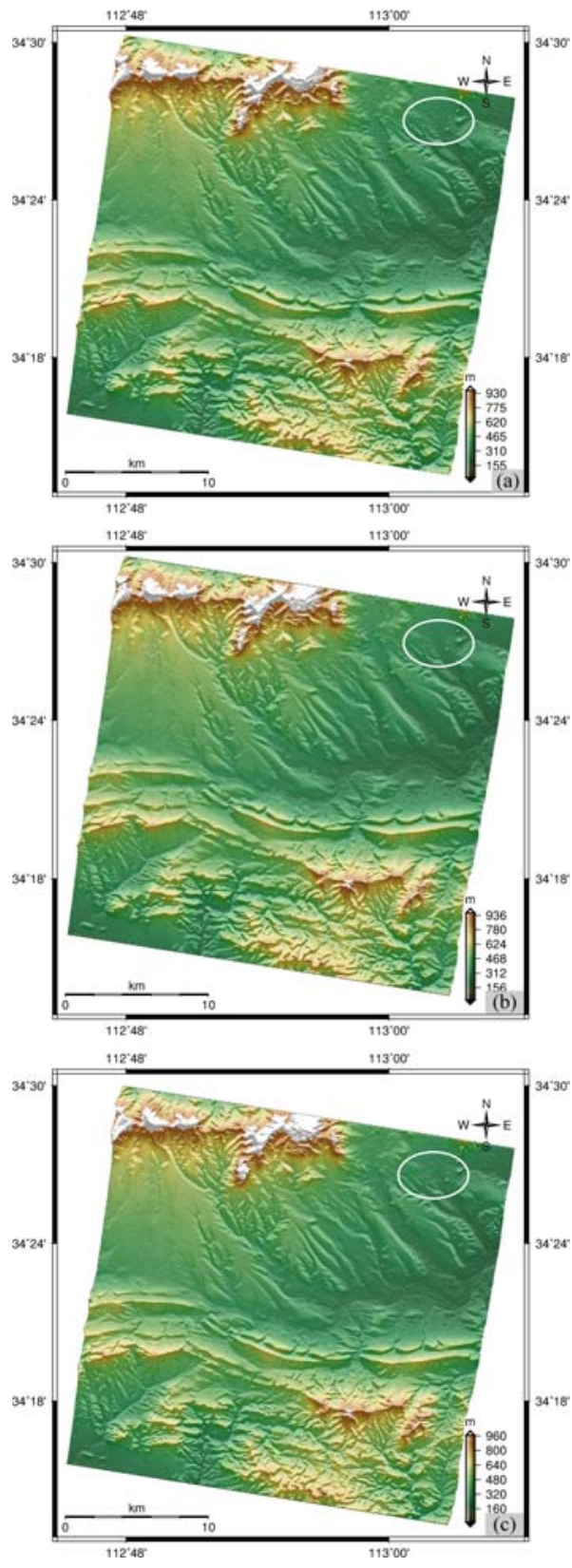

Figure 3. TanDEM-X DEMs obtained using ML factors of (a) $8 \times 8$, (b) $4 \times 4$, and (c) $2 \times 2$. Elliptical region is a residential area. 


\section{CONCLUSION}

Global DEM acquisition is accomplished by NASA and DLR by providing SRTM data and WorldDEM data using InSAR technology. But China does not have interferometric satellite used for surveying and mapping since now. GF-3 was designed for marine resources observation, the interferometric capability was not comprehensively considered. If the recursive baseline is controlled much smaller than the critical baseline, the interferometric applications such as differential InSAR, multitemporal InSAR, and Tomo-SAR can be conducted. If the HoA is furthermore constrained within $35-55 \mathrm{~m}$, and the interferometric parameters such as baseline, interferometric phase, as well as the geometric parameters are improved, the operational DEM acquisition can be accomplished.

In this paper, we use different ML factors to calculate DEM data. In the Laopozhai region of GF-3 interferogram and the whole image of TanDEM-X interferogram, the method provides more consistent DEM when ML factors decrease. Assessment of TanDEM-X data show that the accuracy of DEMs are $9.4 \mathrm{~m}$, $9.3 \mathrm{~m}$, and $8.3 \mathrm{~m}$ for ML factors of 64, 16 and 4, respectively. The proposed method is expected to be used to calculate DEMs with both high accuracy and fine resolution.

\section{ACKNOWLEDGEMENTS}

This research is supported by the National Key R\&D Programme of China (No. 2017YFB0502700), Civilian Space Programme of China (No. D010102), National Basic Surveying and Mapping Science and Technology Plan (No. 2018KJ0204/2018KJ0304).

\section{REFERENCES}

Buckreuß, S., 2016. TerraSAR-X and TanDEM-X mission status, TerraSAR-X/TanDEM-X Science Meeting, Oberpfaffenhofen, Germany.

Gao, X., Liu, Y., Li, T., Wu, D., 2017. High precision DEM generation algorithm based on InSAR multi-look iteration. Remote Sensing 9, 741.

Hueso González, J., Walter Antony, J.M., Bachmann, M., Krieger, G., Zink, M., Schrank, D., Schwerdt, M., 2012. Bistatic system and baseline calibration in TanDEM-X to ensure the global digital elevation model quality. ISPRS Journal of Photogrammetry and Remote Sensing 73, 3-11.

Jiang, M., Li, Z.W., Ding, X.L., Zhu, J.J., Feng, G.C., 2011. Modeling minimum and maximum detectable deformation gradients of interferometric SAR measurements. International Journal of Applied Earth Observation and Geoinformation 13, 766-777.

Montenbruck, O., Wermuth, M., Kahle, R., 2011. GPS based relative navigation for the TanDEM-X mission - First flight results. Naviation: Journal of the Institute of Navigation 58, 293-304.

Rodńguez, E., Morris, C.S., Belz, J.E., Chapin, E.C., Martin, J.M., Daffer, W., Hensley, S., 2005. An assessment of the SRTM topographic products. JPL, p. 143.

Rossi, C., Rodriguez Gonzalez, F., Fritz, T., Yague-Martinez, N., Eineder, M., 2012. TanDEM-X calibrated Raw DEM generation. ISPRS Journal of Photogrammetry and Remote Sensing $73,12-20$. 